\title{
Merger waves: A model of endogenous mergers
}

\author{
Larry D. Qiu and Wen Zhou*
}

November 1, 2006

\begin{abstract}
We develop a model of endogenous mergers to study their dynamic process. Firms choose whether, when, and with whom to merge. Two necessary conditions are identified for mergers to occur: firm heterogeneity and negative demand shocks. We show that mergers are strategic complements and therefore tend to occur in waves. Moreover, some mergers occur for strategic reasons in order to precipitate further mergers.
\end{abstract}

Keywords: endogenous mergers, strategic mergers, merger waves, firm heterogeneity, demand shocks

JEL Code: L13, L41, D43

\section{Introduction}

Mergers have become increasingly widespread in recent years. According to the UN's World Investment Report (UN, 2000), worldwide mergers and acquisitions (M\&A) grew at an annual rate of 42 percent over the period 1980-1999 to reach US\$2.3 trillion in 1999 . More than 24,000 M\&A took place during that period, and the value of M\&A relative to world GDP rose from 0.3 percent in 1980 to 2 percent in 1990 and to 8 percent in 1999. Standard \& Poor's has predicted that consolidation through M\&A would reduce the number of auto companies from 40 in 1998 to about 20 in the 21 st century. ${ }^{1}$

Economic studies of horizontal mergers have focused mainly on two questions: why firms merge, and how they merge. To answer the first question, researchers have typically

\footnotetext{
${ }^{*}$ Qiu and Zhou: Department of Economics, Hong Kong University of Science and Technology, Hong Kong. Corresponding author: Wen Zhou, Tel: (852) 2358-7616; email: wenzhou@ust.hk. We would like to thank Jiahua Che, the Editor Raymond Deneckere and two anonymous referees for their valuable comments and suggestions. We are grateful for financial support from the Research Grants Council of Hong Kong (HKUST6428/05H).

${ }^{1}$ Bill Vlasic, "The first global car colossus" (Business Week, May 18, 1998, p.40).
} 
assumed a single merger, which is decided by a number of designated firms collectively. Non-participants are supposed to remain independent. ${ }^{2}$ Because the merger structure (who merges with whom and who remains independent) is exogenously imposed on the firms, mergers modeled in such a way are called exogenous mergers. To address the second question of how firms merge, the exogenous merger structure must be abandoned and the merger process must be modeled explicitly. In particular, firms must make their merger decisions individually. Mergers that result from such a process are called endogenous mergers. Once firms are allowed to make individual choices, the resulting industry dynamics are greatly enriched, as multiple mergers may occur and firms may merge in response to some other mergers.

In this study, we present a model of endogenous mergers and study how firms merge in a dynamic process. Firms play a two-stage game. In the first stage, mergers occur sequentially in an endogenized order. Each merger is between one proposing firm that is drawn randomly and one target firm that is chosen by the proposer. After a merger is completed, another randomly drawn firm may propose another merger. This process continues until no further mergers occur, which ends the first stage. In the second stage, the surviving firms engage in Cournot competition and receive their payoffs.

We identify two necessary conditions for mergers to occur. Mergers occur only if firms have different marginal costs and the industry has experienced a shock that reduces demand. In Cournot competition, the profitability of any given merger depends on the interaction between two forces: the merging firms internalize the competition between themselves, which benefits them, and the non-merging firms free ride on the reduced competition by competing more aggressively, which hurts the merging firms. If firms are homogeneous with constant marginal costs, Salant et al (1983) have shown that the second force dominates and, therefore, a two-firm merger will never occur. If firms are heterogeneous with different marginal costs, as in our model, merging firms will improve their production efficiency through technology transfer and therefore receive some extra

\footnotetext{
${ }^{2}$ One exception is Deneckere and Davidson (1985), who discussed stable coalition structures, i.e., coalition structures that are immune to further mergers.
} 
benefit. For a given cost differential between the merging firms, the benefit is relatively large if the market size is small. A reduction of the market size, resulting from a negative demand shock, may therefore turn an unprofitable merger into a profitable one and cause the merger to occur.

The extensive-form game that we use allows us to characterize the equilibrium merger strategies, which usually lead to a unique path of mergers. For example, in a four-firm industry, we show that a negative demand shock will lead to a merger between the two firms with intermediate efficiency, followed by another merger between the most and least efficient firms. Indeed, such a pattern can be found in the real world. In 1986, the four largest brands in the U.S. carbonated soft drink industry were Coca-Cola, Pepsi, Seven-up and Dr Pepper, with respective retail sales shares of $37.4 \%, 28.9 \%$, $5.7 \%$ and $4.6 \%$ (White, 1989). In January of that year, Pepsi announced its intention to purchase Seven-up. Three weeks later, Coca-Cola announced its intention to purchase Dr Pepper. Although neither merger materialized due to antitrust objections, ${ }^{3}$ the sequence of announced mergers matches the predictions from our analysis.

We find that mergers are strategic complements in the sense that firms' incentives to merge increase when some other firms also merge. This is because other mergers reduce the number of free riders for a given merger, making the merger more profitable. The complementarity between mergers implies that forward-looking firms may engage in mergers strategically. That is, firms may carry out an otherwise unprofitable merger in order to facilitate some further mergers that might otherwise not occur. We demonstrate the presence of strategic mergers in many cases. Because mergers are strategic complements, they tend to occur together, leading to a merger wave. Hence, our study offers an explanation for the well documented observation that mergers tend to occur in waves, one of the "most consistent empirical features of merger activity over the last century" (Andrade, Mitchell and Stafford, 2001).

\footnotetext{
${ }^{3}$ In June 1986, the US Federal Trade Commission (FTC) decided that these mergers were likely to be anticompetitive and declared its preliminary decision to oppose them. Pepsi and Seven-up immediately canceled their merger plans, but Coca-Cola appealed to the Federal District Court and eventually lost the case.
} 
The predictions of our model are supported by empirical and anecdotal evidence. In a cross-industry empirical study of takeover activity in the 1980s, Mitchell and Mulherin (1996) related many mergers to negative industry shocks: "A shock-driven decline in demand can ... pressure firms to merge ..." (p.197). Dutz (1989) presented evidence of mergers in the steel industry as it faced declining demand. In the popular media, declining demand is often given as one of the major reasons behind some industries' merger waves. Furthermore, industrial analysts often view certain mergers as a response to other mergers in the same industry. Commenting on a merger between two oil companies, Conoco and Philips Petroleum, an article in The Economist magazine (November, $22,2001)$ remarked "It is surely no coincidence that the previous wave of mergers swelled just as oil prices collapsed to around $\$ 10$ a barrel." Completed in 1999 , the $\$ 88$ billion merger between Exxon and Mobil was the largest in U.S. corporate history. That merger was thought to be a response to declining demand and a previous merger between two of the industry's major players. ${ }^{4}$

The growing literature on endogenous mergers is still small. Previous contributions have been made by Kamien and Zang (1990), Barros (1998), Gowrisankaran (1999), Fauli-Oller (2000), and Gowrisankaran and Holmes (2004). ${ }^{5}$ Due to the complexity of the problem, the merger process in most models is endogenized only partially. For example, researchers have restricted the number of firms to three (Barros, 1998) or four (FauliOller, 2000), or assumed that mergers occur in a pre-determined order (Gowrisankaran, 1999; Fauli-Oller, 2000). Our study, by contrast, attempts to model endogenous mergers more completely. We assume an arbitrary number of firms which may differ in their

\footnotetext{
4 "Decreased demand for oil, along with overproduction, caused oil prices to plummet in the late 1990s, ... the biggest firms in the oil industry began seeking large scale consolidation." British Petroleum and Amoco were two of the first firms to pursue such a move in August 1998. A few months later, "Mobil and Exxon began eyeing each other....just a few weeks after the merger's announcement, France's Total SA and Belgium's Petrofina SA joined the consolidation frenzy. In March of 1999, BP Amoco and Atlantic Richfield unveiled their $\$ 25$ billion merger plans." (Hill, 1999, p.415). Similar dynamics can be found in virtually every industry: pharmaceuticals (The Economist, March 27, 2004, p.60), health management (Hill, 1999, p.17), software (The Economist, December 29, 2004), pulp and paper (Hill, 1999, p.258), and automobiles (New York Times, November 14, 1998).

${ }^{5}$ Some researchers have used cooperative games to analyze endogenous mergers (Barros, 1998; Horn and Persson, 2001). For our purpose of analyzing the dynamic process of mergers, the non-cooperative game is the proper analytical tool.
} 
marginal costs, and we endogenize the order of mergers. Although Kamien and Zang (1990) modeled the merger process in a general way, because their firms are identical, they missed the more interesting question of what mergers will occur. They concluded only that full monopolization through mergers will not happen.

Although merger waves prevail in reality, few existing models are sufficiently rich to explain them. By modeling mergers in a simultaneous game, Kamien and Zang (1990) excluded the possibility of strategic mergers and merger waves. While Gowrisankaran (1999) assumed that mergers occur sequentially, his analysis focused on industry dynamics through merger, investment, entry and exit with random returns, rather than on the strategic interaction between mergers. To our knowledge, Fauli-Oller (2000) has provided the only theoretical framework for the study of strategic mergers ${ }^{6}$ but his merger game (two efficient firms take turns to bid for two inefficient firms) is not completely endogenous. We analyze strategic mergers when the merger structure and sequence are both endogenous, thus providing a more realistic setting for the study of merger waves.

The paper is organized as follows. After setting up the model in Section 2, we discuss firm strategies and the profitability of mergers in Section 3. These results are needed in later analysis to derive the equilibria. In Section 4, a four-firm industry is thoroughly analyzed as a special case. We show how to derive the equilibrium. All of the major results in the study, including negative demand shocks as causes of mergers, strategic mergers and merger waves, are present in this special case. Finally, Section 5 presents the results for the general case and discusses their implications. All proofs are provided in the Appendix.

\section{The model}

Consider an industry in which $n(n \geq 3)$ risk-neutral firms produce a homogeneous good. The set of these firms is denoted as $N$. Each firm has a constant marginal cost, $c_{i} \geq 0$ for firm $i(i=1,2, \cdots, n)$, and zero fixed costs. The firms are indexed so that

\footnotetext{
${ }^{6}$ Nilssen and Sorgard (1998) studied the interaction between two sequential exogenous mergers.
} 
$c_{1} \leq c_{2} \leq \cdots \leq c_{n}$. Market demand is given as $p=\alpha-Q$, where $p$ is the market price, $Q$ is the total output of the $n$ firms, and $\alpha$ represents the market size. Assume no entry.

Firms can merge. Assume that any merger is between two firms. Because marginal costs are constant, when two firms merge the merged entity will shut down the high-cost unit and use only the low-cost unit for production. Let $i+j$ stand for the merger between firms $i$ and $j$. Then, the merged entity's marginal cost is equal to $\min \left\{c_{i}, c_{j}\right\}$, or $c_{\min \{i, j\}}$. Therefore, a merger can be viewed as an acquisition of a high-cost firm (hereafter the acquiree) by a low-cost firm (hereafter the acquirer).

The firms engage in a two-stage game. In the first stage, they make merger decisions. Assume that mergers take place sequentially in multiple rounds, one round for each merger. The number of rounds will be determined endogenously. A firm may participate in multiple rounds of mergers as long as it has not been acquired. At the beginning of each round, a firm is drawn randomly from the pool of existing firms. The drawn firm may pass or propose a merger. If it passes, there will be another random draw from the same pool. If the firm proposes (in which case it is called the proposer), it specifies a merger partner (the target) and an acquisition price that is paid by the acquirer to the acquiree. ${ }^{7}$ The target may then accept or reject the proposal. If the target accepts, the merger takes place (i.e., the acquiree leaves with the payment) and the game proceeds to the next round. If the target rejects the proposal, the entire merger stage ends. ${ }^{8}$ If all firms have been drawn and they all choose to pass, the merger stage also ends. In the second stage, all remaining firms carry out Cournot competition and receive their payoffs. We look for the Markov Perfect Equilibria (MPE) of the game, i.e., equilibria in which firms' strategies depend only on payoff-relevant histories of their past actions (Maskin and Tirole, 1989).

Two clarifications are in order. First, we use two distinct pairs of terms to describe the relationships between two firms that merge. In the acquirer-acquiree pair, the acquirer

\footnotetext{
${ }^{7}$ If the proposer is the acquirer, it offers to acquire the target at the price; if the proposer is the acquiree, it asks to be acquired at the price.

${ }^{8}$ We have also analyzed an alternative merger game in which the game continues even when a merger proposal is rejected. We found no change to the equilibrium. See the discussion in the conclusion.
} 
has the lower cost and the acquiree has the higher cost. In the proposer-target pair, the proposer is the firm who makes a merger proposal and the target is the firm which receives the proposal. Depending on their relative costs, both the proposer and the target can be an acquirer or an acquiree. Second, our extensive-form game implies that the proposer captures the entire surplus of a particular merger. That is, the proposer has all the bargaining power ex post. However, since the two merging firms have an equal chance of being the proposer, they have the same bargaining power ex ante.

We make two assumptions.

Assumption 1. $\alpha \geq \alpha_{0} \equiv(n+1) c_{n}-\sum_{i=1}^{n} c_{i}$.

Assumption 2. Mergers can proceed up to a duopoly, but full monopolization is not allowed.

The first assumption ensures that all firms produce non-negative quantities. There are two justifications for the second assumption. On the one hand, it captures the idea that, somewhere down the road in the merger process, the antitrust authority will step in and prevent a merger that will substantially increase the existing firms' market power. On the other hand, if full monopolization is allowed, we can show that, without a trigger, mergers occur spontaneously and the industry invariably ends up with a monopoly. Then the original market structure of $n$ firms is unjustifiable. Note that the cutoff line between monopoly and duopoly is inconsequential. If the line is moved so that the industry must have at least three or four firms, our major conclusions still hold.

For convenience, we list the following notation that is used in the analysis (exact definitions are provided later):

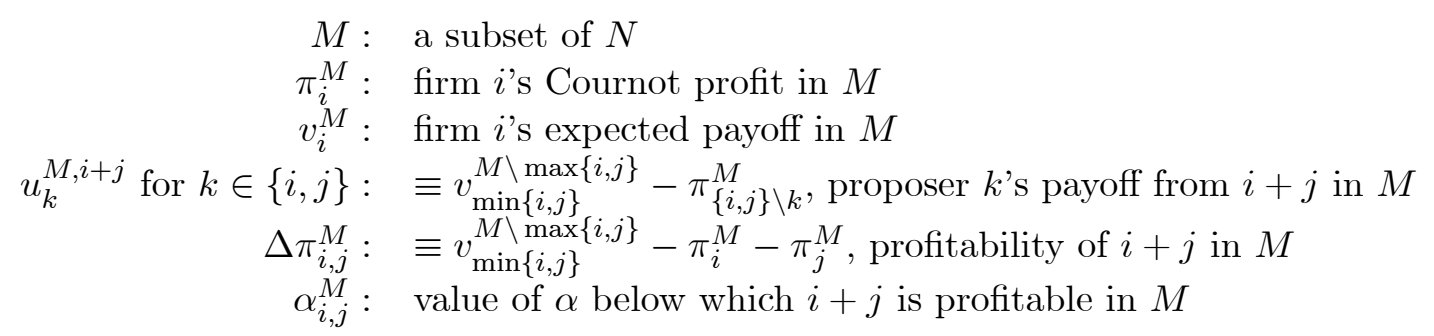




\section{Preliminaries}

In this section, we first derive the second-stage equilibrium for any given merger outcome resulting from the first-stage game. Then, we move back to the first stage to analyze firms' strategies and the profitability of the mergers. The analysis serves as the stepping stone for us to derive the equilibrium of the whole game.

\section{The second-stage: Cournot profits}

Consider a set, $M \subseteq N$, of firms that engage in Cournot competition. We can easily obtain firm $i$ 's $(i \in M)$ Cournot quantity and profit as

$$
q_{i}^{M}=\frac{\alpha+C_{M}}{m+1}-c_{i} \text { and } \pi_{i}^{M}=\left(q_{i}^{M}\right)^{2}=\left(\frac{\alpha+C_{M}}{m+1}-c_{i}\right)^{2}
$$

where $m \equiv|M|$ is the number of firms in $M$ and $C_{M}=\sum_{j \in M} c_{j}$. Immediately, we have: Lemma 1. $\pi_{i}^{M^{\prime}}>\pi_{i}^{M}$ for all $i \in M^{\prime} \subset M$.

Lemma 1 implies that a firm benefits from mergers among its competitors, a conclusion known in the literature as the free-riding effect of mergers (Salant et al., 1983).

\section{The first-stage: Strategies and merger profitability}

Given a set, $M$, of firms, let $v_{i}^{M}$ denote firm $i$ 's $(i \in M)$ expected payoff in $M$. If there is no merger in $M, v_{i}^{M}=\pi_{i}^{M}$. If, in a merger proposal, $i$ acquires $j$ at acquisition price $T$, then, conditional on the proposal being accepted, $v_{i}^{M}=v_{i}^{M \backslash j}-T, v_{j}^{M}=T$ and $v_{k}^{M}=v_{k}^{M \backslash j}$ for $k \in M \backslash\{i, j\}$. As we will see, $v_{i}^{M}$ and $\pi_{i}^{M}$ are two important values that determine $i$ 's optimal strategies in the merger stage.

A firm needs to make a decision when it is drawn or when it is a target. A drawn firm chooses between "pass" and "propose a merger" and, in the latter case, chooses the target and the acquisition price. A target chooses between "accept" and "reject".

\section{Target's optimal strategy and proposer's acquisition price}

Lemma 2. If $i$ proposes acquiring $j$ at acquisition price $T$, then,

(a) $j$ accepts the proposal if and only if $T \geq \pi_{j}^{M}$ for $i<j$ and $T \leq v_{j}^{M \backslash i}-\pi_{j}^{M}$ for $i>j$. 
(b) $i$ 's optimal acquisition price is $T=\pi_{j}^{M}$ for $i<j$ and $T=v_{j}^{M \backslash i}-\pi_{j}^{M}$ for $i>j$.

Therefore, once a target, say, $j$, is chosen, the acquisition price will be such that the target's expected payoff, regardless of whether it is the acquirer or acquiree, is $\pi_{j}^{M}$, and the target will accept the proposal. As a result, the proposer, say, $i$, has a payoff $u_{i}^{M, i+j}=v_{\min \{i, j\}}^{M \backslash \max \{i, j\}}-\pi_{j}^{M}$. From now on, we focus on the proposer's other choices; namely whether to pass or propose and, in the latter case, to whom.

\section{Proposer's preferences}

Based on Lemmas 1 and 2, we have $v_{i}^{M} \geq \pi_{i}^{M}$ for any $i \in M$. The following two lemmas state a proposer's preference.

Lemma 3. For $i, j \in M$ and $i<j, u_{i}^{M, i+j}<v_{i}^{M \backslash j}$.

Lemma 4. For $i, j, k \in M$ and $i<j<k$, if there is no further merger,

(a) $u_{k}^{M, i+k}>u_{k}^{M, j+k}$;

(b) $u_{i}^{M, i+k}>u_{i}^{M, i+j}$ if and only if $m\left(q_{j}^{M}+q_{k}^{M}\right)>\left(q_{i}^{M \backslash j}+q_{i}^{M \backslash k}\right)$.

Lemma 3 states that a proposer prefers passing if its intended acquiree will be acquired by a third firm. Lemma 4 indicates a proposer's optimal choice of target when there is no further merger: if the proposer seeks to be acquired, its target should be the most efficient firm in $M$; if the proposer seeks to acquire some firm, its target should generally be the least efficient firm in $M .^{9}$ The intuition for Lemma 4 is the following. When a firm $(k)$ is acquired, regardless of who the acquirer is, the market structure after the merger is the same: firm $k$ drops out and market competition is reduced. A more efficient acquirer, however, benefits more from the reduced competition and therefore generates more surplus to the merging firms. When a firm $(i)$ proposes acquiring another firm, the market structure after the merger depends on who the acquiree is. A more efficient acquiree means the elimination of a stronger rival and thus a greater reduction of competition. However, $i$ needs to pay more for the elimination because the acquiree's pre-merger profit is also higher. In Cournot competition, the acquirer pays

\footnotetext{
${ }^{9}$ The condition for (b) is generally satisfied unless both acquirees are extremely weak and there are very few firms in $M$.
} 
the full cost of eliminating a rival but receives only a fraction of the benefit of the reduced competition (the free-riding effect). It turns out that the merger is generally more profitable when the acquiree is less efficient.

\section{Merger profitability}

Denote $\Delta \pi_{i, j}^{M} \equiv v_{\min \{i, j\}}^{M \backslash \max \{i, j\}}-\pi_{i}^{M}-\pi_{j}^{M}$ as the profitability of $i+j$ in $M$. The merger is said to be profitable if and only if $\Delta \pi_{i, j}^{M} \geq 0$. An unprofitable merger will never take place because neither of the two firms will propose it; passing is a better choice: $v_{i}^{M} \geq \pi_{i}^{M}>v_{\min \{i, j\}}^{M \backslash \max \{i, j\}}-\pi_{j}^{M}=u_{i}^{M, i+j}$ and similarly for firm $j$. Profitability is therefore a necessary condition for a merger to take place. The following lemma gives a sufficient condition.

Lemma 5. Given $M$, if $i+j$ is the only profitable merger, then there is a unique MPE in which (when drawn to move) $i$ proposes to $j, j$ proposes to $i$, and all other firms pass. The MPE results in a unique equilibrium merger of $i+j$.

For a merger to occur as the unique equilibrium, a sufficient condition is that the merger is the only profitable merger in $M$. This condition is not necessary. Even if there exist multiple profitable mergers, a merger $i+j$ may still be the unique equilibrium. This will be the case if the equilibrium strategies are such that $i$ and $j$ propose to each other while all other firms pass.

If $i+j$ is the unique equilibrium merger in $M$, Lemma 2 implies that $i$ receives $u_{i}^{M, i+j}$ (when $i$ proposes to $j$ ) with probability $\frac{1}{2}$ and $\pi_{i}^{M}$ (when $j$ proposes to $i$ ) with probability $\frac{1}{2}$. Hence, $i$ 's expected payoff is

$$
v_{i}^{M}=\frac{1}{2}\left(v_{\min \{i, j\}}^{M \backslash \max \{i, j\}}-\pi_{j}^{M}\right)+\frac{1}{2} \pi_{i}^{M}=\pi_{i}^{M}+\frac{1}{2} \Delta \pi_{i, j}^{M}
$$

Similarly, $v_{j}^{M}=\pi_{j}^{M}+\frac{1}{2} \Delta \pi_{i, j}^{M}$. That is, for a unique equilibrium merger, the two merging firms expect to split the merger surplus equally.

We now state the market condition for a profitable merger.

Lemma 6. Consider a possible merger between $i$ and $j(i<j)$ in $M$ with $m \geq 3$ and 
assume no further mergers. Then, $i+j$ is profitable if and only if

$$
\alpha \leq \frac{(m+1)}{(m-1)^{2}-2}\left\{\left(m^{2}-1\right) c_{j}-2 m c_{i}\right\}-C_{M} \equiv \alpha_{i, j}^{M}
$$

Notice that the condition is never satisfied if all costs are identical: $\alpha_{i, j}^{M}=\alpha_{0}=c$ if $c_{k}=c$ for $k \in M$. Merging firms reduce their competition in order to raise their joint profits. The reduced competition, however, induces non-merging firms to expand, which hurts the merged firms. ${ }^{10}$ If costs are identical, the damage from non-merging firms' expansion dominates and the merger is never profitable (Salant et al, 1983). If costs are different, the merging firms gain some extra benefit from the improved production efficiency. ${ }^{11}$ Since costs are measured relative to the market size, when $\alpha$ is small, the efficiency improvement from a given cost differential is substantial and the merger becomes profitable.

\section{Special cases}

Lemmas 1-6 help us derive the firms' equilibrium strategies that result in an equilibrium merger configuration, i.e., a series of mergers in a particular order. In this section, we demonstrate how this can be done through the special case of $n=4$. The analysis allows us to see some interesting features of mergers such as strategic mergers and merger waves. It also shows how mergers may be triggered by demand shocks.

The general procedure of deriving the equilibrium is the following. List all possible merger configurations. For every configuration, find the range of $\alpha$ in which the configuration is profitable, i.e., every merger in the configuration is profitable. Since the last merger in any given configuration is profitable only when $\alpha$ is below a critical value (Lemma 6), the range of $\alpha$ for a profitable configuration will necessarily have an upper bound (and may also have a lower bound). Put together the configurations and the

\footnotetext{
${ }^{10}$ If firms compete in prices, the reaction from non-merging firms will benefit the merged firms (Deneckere and Davidson, 1985).

${ }^{11}$ Production efficiency is improved because the merged firms can produce any joint output at a cost lower than that without the merger. We can think of the acquiree as adopting the more-advanced technology from the acquirer.
} 
associated $\alpha$-ranges. Within a range, if there is only one profitable configuration, it will be the unique equilibrium (Lemma 5). If there are several profitable configurations, we need to further analyze each active firm's preference among profitable mergers in each round using Lemmas 3 and 4 (see the Appendix for details in the specific cases of $n=3$, 5 and 6 ). For any $\alpha \geq \alpha_{0}$, such an analysis will lead to a unique MPE, from which the equilibrium merger configurations can be derived. ${ }^{12}$

Now consider the case of $N=\{1,2,3,4\}$ with $c_{i}=i-1$ for $i \in N$. Then, $\alpha_{0}=9$. By Assumption 2, a configuration can have at most two mergers. For configurations that contain a single merger (there are six of them), Lemma 6 applies and the critical value is $\alpha_{i, j}^{N}=\frac{5}{7}\left(15 c_{j}-8 c_{i}\right)-6$. The highest $\alpha_{i, j}^{N}$ is for $i=1$ and $j=4$ with $\alpha_{1,4}^{N}=26.1$. Therefore, when $\alpha>26.1$, there is no equilibrium that contains a single merger.

Consider configurations that contain two sequential mergers. The acquiree in the first round must be firm 2, 3 or 4 . If firm 2 is acquired (by firm 1) in the first round, we can show (see similar analysis for $n=3$ in the Appendix) that the remaining firms will have a further merger of $1+4$ for $19<\alpha \leq 43$ and no merger for $\alpha>43$. Given the second-round merger, however, the first-round merger, $1+2$, is unprofitable for $\alpha>19$. If firm 3 is acquired (by 1 or 2 ) in the first round, we can again show that the remaining firms will have a further merger of $1+4$ for $12<\alpha \leq 44$ (and no merger for $\alpha>44$ ). This implies two possible merger configurations: $1+3 \Rightarrow 1+4$ (i.e., $1+3$ followed by $1+4)$ and $2+3 \Rightarrow 1+4$. We find that the first-round merger $1+3$ is unprofitable for $\alpha>22.2$, while $2+3$ is profitable for any $\alpha \geq \alpha_{0}$. Finally, if firm 4 is acquired in the first round, we can show (see the proof for the case of $n=3$ in the Appendix) that the remaining firms will not merge when $\alpha>29$.

In summary, for $\alpha \in(29,44], 2+3 \Rightarrow 1+4$ is the only profitable configuration. By Lemma 5, there is a unique MPE: in the first round, firms 2 and 3 propose to each other while firms 1 and 4 pass; in the second round, firms 1 and 4 propose to each other while

\footnotetext{
${ }^{12}$ A unique MPE may or may not result in a unique merger configuration. For example, if all firms pass in an equilibrium except $i$ and $j$ which propose to each other, the resulting configuration is uniquely $i+j$. If, on the other hand, $i$ proposes to $j$ while both $j$ and $k$ propose to $i$, the resulting configuration is $i+j$ with probability $\frac{2}{3}$ and $i+k$ with probability $\frac{1}{3}$.
} 


\begin{tabular}{|c|c|l|}
\hline$N$ & Range of $\alpha$ & \multicolumn{1}{|c|}{ Equilibrium Merger Configuration(s) } \\
\hline \multirow{3}{*}{$1,2,3$} & $(29, \infty)$ & No merger \\
\cline { 2 - 3 } & $(6.9,29]$ & $1+3$ \\
\cline { 2 - 3 } & $(6.6,6.9]$ & $1+3$ with prob. $\frac{2}{3}$ and $1+2$ with prob. $\frac{1}{3}$ \\
\cline { 2 - 3 }$\{1,2,3,4\}$ & $(5,6.6]$ & $1+2$ \\
\cline { 2 - 3 } & $(44, \infty)$ & No merger \\
\hline \multirow{2}{*}{$1,2, \cdots, 5$} & $(59,44]$ & $2+3 \Rightarrow 1+4$ \\
\cline { 2 - 3 } & $(44,59]$ & No merger \\
\hline \multirow{2}{*}{$1,2, \cdots, 6$} & $(74, \infty)$ & No merger \\
\cline { 2 - 3 } & Slightly below 74 & $\begin{array}{l}2+i \Rightarrow 1+j \Rightarrow 2+3 \Rightarrow 1+6, \text { in which } \\
i=4 \text { with prob. } \frac{1}{3}, i=5 \text { with prob. } \frac{2}{3}, \text { and } j=\{4,5\} \backslash i\end{array}$ \\
\hline
\end{tabular}

Table 1: Equilibrium Merger Configurations for $\mathrm{n}=3,4,5,6$.

firm 2 passes. The MPE results in a unique merger configuration: $2+3 \Rightarrow 1+4$.

A similar (albeit much more complicated) analysis can be used to derive the equilibrium for $\alpha \in\left[\alpha_{0}, 29\right]$, which shows that mergers always occur in this range. On the other hand, the above analysis has revealed that no merger occurs for $\alpha>44$. In fact, this pattern exists for any general $n$ : there exists a critical value of $\alpha$ such that mergers occur if and only if $\alpha$ is below the level.

The relationship between the two mergers in the equilibrium can be found by comparing the critical values of $\alpha$ for various merger configurations. It turns out that $2+3$ is unprofitable unless 1 and 4 merge subsequently. Conversely, $1+4$ is unprofitable unless 2 and 3 have merged. We can show that firms' incentives to merge increase when other firms also merge. Therefore, mergers are strategic complements, which immediately implies that mergers tend to occur together, leading to a merger wave. Furthermore, in the example's wave of mergers, the first merger is strategic because, given it is unprofitable, it serves the explicit purpose of inducing a further merger that will otherwise not occur.

So far we have analyzed the case of $n=4$. A similar analysis can be applied to $n=3,5$, and 6 (with $c_{i}=i-1$ for $i \in N$ ), and we report in Table 1 the equilibrium merger configurations of these cases. For $n=3$, we have a complete characterization of equilibrium merger configurations for $\alpha \geq \alpha_{0}$; for $n>3$, we only report the equilibrium merger configurations for a subset of $\alpha$, as the complexity of the analysis increases with $n$ 
exponentially. The analyses and results reveal a common feature in all cases: mergers are caused by negative demand shocks, they occur in waves, and some mergers are strategic.

\section{The general case}

Return now to the general case with $n$ firms and general costs. Define

$$
\alpha(k)=f(k) c_{n}-g(k) c_{1}-\sum_{i=1}^{n-k} c_{i}
$$

where $k \in\{1,2, \cdots, n-2\}, f(k)=\frac{(n-k+1)^{3}+n-k+1}{(n-k)^{2}-2}$ and $g(k)=\frac{2(n-k)^{2}+6 n-6 k+4}{(n-k)^{2}-2}$. As shown in the Appendix, $\alpha(k)$ is the value of $\alpha$ below which exactly $k$ mergers occur in $N$. Let $\alpha^{*}$ be the maximal value of $\alpha$ and $k^{*}$ be the maximizer. We now state the main result of the study.

Proposition. A necessary condition for any merger to occur is $\alpha<\alpha^{*}$. If mergers occur when $\alpha$ is slightly below $\alpha^{*}$, the merger configuration(s) must have the following features:

(a) there are $k^{*}$ rounds of mergers;

(b) the $k^{*}$ least efficient firms are acquired; and

(c) the last-round merger is between firm 1 and firm $n$.

The Proposition provides a necessary condition for mergers. Firms start to merge only when the market size, $\alpha$, is below a critical level. The Proposition also characterizes some properties of the mergers for $\alpha$ slightly below the level. In the rest of this section, we discuss the applications and implications of the Proposition.

\section{- The role of demand shocks and firm heterogeneity}

A model of endogenous mergers needs to explain not only how firms merge, but also what triggers the mergers. We have used the special cases in the previous section to demonstrate how firms merge. Now the Proposition identifies one factor that may trigger mergers: firms start to merge only when the demand is below a critical level. Since the original market structure with $n$ firms (i.e., no firms merge) must also be an equilibrium, the demand must be above the critical level originally. Mergers therefore 
must have been caused by a drop in the demand. In other words, mergers are caused by a negative demand shock.

Firm heterogeneity also plays an important role in mergers. If firms are identical, i.e., $c_{i}=c$ for $i \in N$, we have $\alpha_{0}=c$ and $\alpha(k)=c$ for all $k \in[1, n-2]$, and the necessary condition in the Proposition is never satisfied. Thus, firms will never merge in response to any demand shock if they all have identical, constant marginal costs. This result has also been obtained in other endogenous merger models in which firms move simultaneously (Kamien and Zang, 1990; Thoron, 1998). Our result is stronger because we show that firms do not merge even when they move sequentially. Indeed, we show that firms will merge if their costs are sufficiently different.

\section{- Strategic mergers and merger waves}

An intriguing feature of the equilibrium characterized in the Proposition is that a demand shock may cause multiple mergers $\left(k^{*} \geq 2\right)$ in sequence, i.e., a merger wave. In some waves of mergers, each individual merger is an independent response to the shock. In other waves, mergers are interdependent, i.e., some mergers occur because some other mergers have already occurred. The latter case is particularly interesting and deserves further discussion. Recall that free-riding by non-merging firms reduces the profitability of a given merger. If the number of free riders is reduced, an otherwise unprofitable merger may become profitable. Between two potential mergers, if one merger occurs, the number of firms that free ride on the second merger is reduced, making the second merger more likely to occur. Therefore, firms' incentives to merge increase when other mergers also occur. Mergers are strategic complements and, as a result, tend to occur in waves.

Whether or not a demand shock will cause a merger wave depends mainly on the number of firms in the industry. By assigning costs to the $n$ firms, we can calculate the values of $\alpha(k)$ and determine $k^{*}$. Our numerical analysis shows that, ${ }^{13}$ typically,

\footnotetext{
${ }^{13}$ If $c_{i}=i-1$ for $i \in N$, we find that $\alpha(k)$ increases with $k$ when $n<9$ (which means $\left.k^{*}=n-2\right)$ and is U-shaped when $n>9$ (which means $k^{*}$ is either 1 or $\left.n-2\right)$. In the latter case, $\alpha(1)<\alpha(n-2)$ for $n \leq 23\left(\right.$ so $\left.k^{*}=n-2\right)$ and $\alpha(1)>\alpha(n-2)$ for $n>23\left(\right.$ so $\left.k^{*}=1\right)$.
} 
$k^{*}=n-2$ for $n \leq 23$ and $k^{*}=1$ for $n>23$. Therefore, mergers tend to occur in waves when $n$ is small.

In our model, mergers occur sequentially. The complementarity between mergers then opens the possibility for strategic behavior. Consider two potential mergers, $A$ followed by $B$. Neither is profitable by itself. But if $A$ has occurred, $B$ becomes profitable and will therefore occur. Given that $B$ will occur subsequently, $A$ becomes profitable as well. In that case, $A$ will be carried out. This merger is strategic because, although unprofitable by itself, the merger nevertheless occurs in order to induce further mergers that might otherwise not occur. An example of such a strategic merger has been demonstrated earlier in the case of $n=4$.

\section{- Pattern of the equilibrium merger configuration}

The exact path of equilibrium mergers depends on the firms' costs. In order to search for some common features of the equilibrium merger path, we conduct a numerical simulation, in which we assign random costs to the $n$ firms and derive the corresponding equilibrium configurations. A general pattern can be observed. The acquired firms are generally acquired in the order of $n-1, n-2, \cdots, n-\left(k^{*}-1\right)$, and finally $n$. If $k^{*}=n-2$ (i.e., the final market is a duopoly), then firms 1 and 2 take turns to acquire the other $n-2$ firms. If $k^{*} \neq n-2, k^{*}$ is usually very small $\left(k^{*}<\frac{n}{2}\right)$. Then, the first $k^{*}$ firms in $N$, in the order $k^{*}, k^{*}-1, \cdots, 2,1$, take turns to do the acquiring. Notice that a firm does not participate in any two consecutive mergers and a firm does not acquire any other firm before itself being acquired.

\section{口 Production shocks}

The discussion so far has focused on demand shocks as a cause for mergers. In fact, the model can accommodate other causes. Recall from the Proposition that the condition for mergers to take place is simply $\alpha$ being lower than $\alpha^{*}$, which is a linear combination

For general values of the costs, we conduct a numerical simulation by assigning random costs to the $n$ firms repeatedly. The results indicate that the shape of $\alpha(k)$ is not sensitive to the cost distribution and therefore the above pattern about $k^{*}$ (i.e., $k^{*}=n-2$ for small $n$ and $k^{*}=1$ otherwise) continues to hold.

It is also possible that $k^{*}$ is greater than 1 (but smaller than $\frac{n}{2}$ ). This happens for some special values of the costs. 
of the firms' costs. This condition may be satisfied if $\alpha$ drops while $\alpha^{*}$ does not change, which corresponds to a demand shock. The condition may also be satisfied if $\alpha$ does not change but $\alpha^{*}$ increases. This would be the case if there is a negative supply shock that increases some or all firms' costs. Hence, regardless of demand or supply shocks, firms start to merge only when business becomes tougher. The conclusion that negative supply shocks may trigger mergers is also supported by empirical evidence. ${ }^{14}$

\section{Concluding remarks}

In this study, we present a model of endogenous mergers and analyze their dynamic process. The equilibrium demonstrates that a negative demand shock may cause a merger wave and that some mergers may occur for strategic reasons.

Although entry and exit are assumed away in our model, they can be incorporated. As is evident from the expression of $\alpha^{*}$, exit of a firm reduces $n$, which tends to lower $\alpha^{*}$. As a result, the necessary condition $\left(\alpha<\alpha^{*}\right)$ is further relaxed and mergers will not occur. On the other hand, entry by one or several new firms may make it more or less likely for the condition to be satisfied. ${ }^{15}$ Thus, we conjecture that the exit of existing firms tends to stabilize the market structure (i.e., make mergers less likely), while the entry of new firms has the potential to change the market structure dramatically through a merger wave.

Demand and costs are assumed to be linear mainly for tractability. As has been pointed out by Perry and Porter (1985) and Cheung (1992), relaxing these assumptions may affect some quantitative results in exogenous mergers, where the equilibrium is derived by comparing firms' profits before and after a designated merger. In endogenous mergers, however, the comparison mainly focuses on each firm's profit from merging with

\footnotetext{
${ }^{14}$ Jensen (1993) suggested that the consolidation in many industries can be traced to increased input prices caused by the oil shocks of the 1970s. The Economist (November, 22, 2001) remarked: "Thanks to the rising cost and risk of exploration in ever more remote areas, life has got harder for oil companies. That explains the wave of consolidation of the past three years that has seen Exxon take over Mobil, BP buy Amoco and Arco, Total absorb Petrofina and Elf Aquitaine, and Chevron buy Texaco."

${ }^{15}$ For example, in $N=\{1,2,3,4\}$, entry by a firm whose cost is below $c_{2}$ or greater than $c_{4}$ may trigger mergers, while exit by firm 3 or entry by one or more firms whose costs are between $c_{2}$ and $c_{4}$ will not trigger any mergers.
} 
different partners. This comparison is less sensitive to the linearity assumptions, so the qualitative conclusions of this study probably will not change when general demand and cost functions are used. Nevertheless, it would be interesting to demonstrate this.

In our model, we assume that the merger stage ends whenever a merger proposal is rejected. This assumption seems strong. Nevertheless, our results should continue to hold when the assumption is relaxed. We have analyzed an alternative game, in which a rejection does not end the merger stage and another proposer will be chosen among the remaining firms. In the cases of $n=3,4,5$ and 6 , we can show that the equilibrium merger configurations are the same as in our original model. Following the analysis of these cases, we argue that the same result holds for a general $n$. Detailed proofs are available on the authors' website. ${ }^{16}$

\section{Appendix}

The appendix provides the proof of all the results in the text. The following notations are used in the proofs:

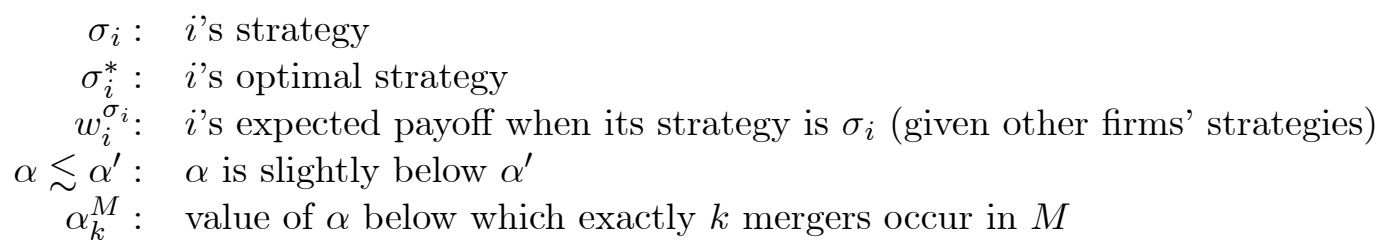

\section{Lemma 1}

Suppose $M \backslash M^{\prime}=\{j\}$. Then, for all $i \in M^{\prime}, q_{i}^{M}=\frac{\alpha+C_{M}}{m+1}-c_{i}<\frac{\alpha+C_{M}-c_{j}}{m}-c_{i}=q_{i}^{M^{\prime}}$ because $\frac{\alpha+C_{M}}{m+1}-c_{j}=q_{j}^{M}>0$. Thus, $\pi_{i}^{M}<\pi_{i}^{M^{\prime}}$. If $M \backslash M^{\prime}$ contains more than one firm, the same inequality continues to hold because $M^{\prime}$ can be reached by consecutively removing firms, one at a time, from $M$.

\section{Lemma 2}

(a) If $j$ rejects the proposal, the merger stage ends and $j$ 's payoff is $\pi_{j}^{M}$. If $j$ accepts the offer, $j$ 's payoff is $T$ if $j$ is the acquiree $(i<j)$ and $v_{j}^{M \backslash i}-T$ if $j$ is the acquirer $(i>j)$. Hence the conclusion.

\footnotetext{
${ }^{16}$ The address is http://ihome.ust.hk/ wenzhou/supplement(Qiu \& Zhou).pdf.
} 
(b) Conditional on $j$ accepting the offer, firm $i$ sets $T$ as low as possible if $i$ is the acquirer $(i<j)$, so $T=\pi_{j}^{M}$. If $i$ is the acquiree $(i>j)$, firm $i$ sets $T$ as high as possible, so $T=v_{j}^{M \backslash i}-\pi_{j}^{M}$.

\section{Lemma 3}

$$
u_{i}^{M, i+j}=v_{i}^{M \backslash j}-\pi_{j}^{M}<v_{i}^{M \backslash j} .
$$

\section{Lemma 4}

(a) $u_{k}^{M, i+k}=\pi_{i}^{M \backslash k}-\pi_{i}^{M}=\left(\frac{\alpha+C_{M}-c_{k}}{m}-c_{i}\right)^{2}-\left(\frac{\alpha+C_{M}}{m+1}-c_{i}\right)^{2}$. Then, keeping $C_{M}$ constant, ${ }^{17} \frac{\partial u_{k}^{M, i+k}}{\partial c_{i}}=2 q_{i}^{M}-2 q_{i}^{M \backslash k}$, which is negative by Lemma 1. This means $u_{k}^{M, i+k}>$ $u_{k}^{M, j+k}$ for $i<j$.

(b) Note

$$
\begin{aligned}
u_{i}^{M, i+k}-u_{i}^{M, i+j} & =\left(\pi_{i}^{M \backslash k}-\pi_{k}^{M}\right)-\left(\pi_{i}^{M \backslash j}-\pi_{j}^{M}\right) \\
& =\left(\pi_{j}^{M}-\pi_{k}^{M}\right)-\left(\pi_{i}^{M \backslash j}-\pi_{i}^{M \backslash k}\right) \\
& =\left(c_{k}-c_{j}\right)\left\{\left(q_{j}^{M}+q_{k}^{M}\right)-\frac{1}{m}\left(q_{i}^{M \backslash j}+q_{i}^{M \backslash k}\right)\right\} .
\end{aligned}
$$

\section{Lemma 5}

Because $i+j$ is the only profitable merger in $M$, for $k \in M \backslash\{i, j\}$, its optimal strategy is "pass", denoted as P. Firm $i$ 's strategy set includes "pass" and "propose to $j$ ", denoted as $R(j)$ and so $\sigma_{i} \in\{P, R(j)\}$. Similarly, $\sigma_{j} \in\{P, R(i)\}$. If $\sigma_{j}=P$, $i$ 's

expected payoff is $\pi_{i}^{M}$ if $\sigma_{i}=P$, denoted as $w_{i}^{P}=\pi_{i}^{M}$. Similarly, $w_{i}^{R(j)}=u_{i}^{M, i+j}$, which is greater than $\pi_{i}^{M}$ by $\Delta \pi_{i, j}^{M}>0$, so $\sigma_{i}^{*}=R(j)$. If, on the other hand, $\sigma_{j}=R(i)$, we have $w_{i}^{P}=\pi_{i}^{M}$ and $w_{i}^{R(j)}=\frac{1}{2}\left(\pi_{i}^{M}+u_{i}^{M, i+j}\right)>\pi_{i}^{M}$, so again $\sigma_{i}^{*}=R(j)$. That is, proposing to $j$ is $i$ 's dominant strategy. Similarly, proposing to $i$ is $j$ 's dominant strategy.

\section{Lemma 6}

If there is no further merger, $v_{\min \{i, j\}}^{M \backslash \max \{i, j\}}=\pi_{i}^{M \backslash j}$. Using the Cournot profits,

$$
\Delta \pi_{i, j}^{M}=\pi_{i}^{M \backslash j}-\pi_{i}^{M}-\pi_{j}^{M}=\frac{q_{j}^{M}}{m^{2}(m+1)} \theta
$$

where $\theta=(m-1)(m+1)^{2} c_{j}-2 m(m+1) c_{i}-\left(\alpha+C_{M}\right)\left(m^{2}-2 m-1\right)$. As a result, $\Delta \pi_{i, j}^{M} \geq 0$ if and only if $\theta \geq 0$, which is equivalent to $\alpha \leq \alpha_{i, j}^{M}$.

\footnotetext{
${ }^{17}$ Our purpose is to compare $u_{k}^{M, i+k}$ with $u_{k}^{M, j+k}$, which is accomplished by switching $i$ and $j$ in $M$. Therefore, $C_{M}$ remains constant when $c_{i}$ changes.
} 


\section{Three firms}

$N=\{1,2,3\}$ and $\alpha_{0}=5$. By Assumption 2, there can be at most one merger, which may be $1+2,1+3$ or $2+3$. From Lemma $6, \alpha_{1,2}^{N}=13, \alpha_{1,3}^{N}=29, \alpha_{2,3}^{N}=17$. For $17<\alpha \leq 29,1+3$ is the only profitable merger and therefore the unique equilibrium (Lemma 5).

For $13<\alpha \leq 17$, there are two profitable mergers: $1+3$ and $2+3$. Both involve firm 3. By Lemma $4(\mathrm{a}), u_{3}^{N, 1+3}>u_{3}^{N, 2+3}$, so $\sigma_{3}^{*}=R(1) . \sigma_{1}, \sigma_{2} \in\{P, R(3)\}$. By Lemma 3 , $v_{1}^{N \backslash 3}>u_{1}^{N, 1+3}$ and $v_{2}^{N \backslash 3}>u_{2}^{N, 2+3}$. Consider firm 2's optimal strategy. If $\sigma_{1}=R(3)$, $w_{2}^{P}=v_{2}^{N \backslash 3}$ and $w_{2}^{R(3)}=\frac{1}{3} u_{2}^{N, 2+3}+\frac{2}{3} v_{2}^{N \backslash 3}<w_{2}^{P}$, so $\sigma_{2}^{*}=P$. If $\sigma_{1}=P, w_{2}^{P}=v_{2}^{N \backslash 3}$ and $w_{2}^{R(3)}=\frac{1}{2} u_{2}^{N, 2+3}+\frac{1}{2} v_{2}^{N \backslash 3}<w_{2}^{P}$, so $\sigma_{2}^{*}=P$. Therefore, $\sigma_{2}^{*}=P$ regardless of $\sigma_{1}$. Given this, $w_{1}^{P}=\pi_{1}^{N}$ and $w_{1}^{R(3)}=u_{1}^{N, 1+3}>\pi_{1}^{N}$, so $\sigma_{1}^{*}=R(3)$. The strategy profile of the three firms, $(R(3), P, R(1))$, leads to a unique equilibrium merger of $1+3$.

For $\alpha \leq 13$, all three mergers are profitable. $v_{3}^{N \backslash 2}>u_{3}^{N, 1+3}>u_{3}^{N, 2+3}>\pi_{3}^{N}$, so $\sigma_{3} \in\{P, R(1)\}$. If $\frac{38+8 \sqrt{10}}{9} \approx 6.9<\alpha \leq 13, v_{1}^{N \backslash 3}>u_{1}^{N, 1+3}>u_{1}^{N, 1+2}$ and $v_{2}^{N \backslash 3}>$ $u_{2}^{N, 1+2}>u_{2}^{N, 2+3}$, so $\sigma_{1} \in\{P, R(3)\}$ and $\sigma_{2} \in\{P, R(1)\}$. Given 2 and 3's possible strategies, $w_{1}^{P}=\pi_{1}^{N}$ and $w_{1}^{R(3)}>\pi_{1}^{N}$, so $\sigma_{1}^{*}=R(3)$. Given $\sigma_{1}^{*}, \sigma_{2}^{*}=P$ and $\sigma_{3}^{*}=R(1)$. The unique equilibrium is $1+3$.

When $\alpha \leq 6.9, u_{2}^{N, 1+2}>\max \left\{v_{2}^{N \backslash 3}, u_{2}^{N, 2+3}\right\}$, so $\sigma_{2}^{*}=R(1)$.

For $\frac{33}{5}<\alpha \leq 6.9, v_{1}^{N \backslash 3}>u_{1}^{N, 1+3}>u_{1}^{N, 1+2}$. Given $\sigma_{2}^{*}$, we have $\sigma_{1}^{*}=R(3)$ regardless of $\sigma_{3}$. Given $\sigma_{1}^{*}$ and $\sigma_{2}^{*}, w_{3}^{P}=\frac{1}{2}\left(\pi_{3}^{N}+v_{3}^{N \backslash 2}\right)$ and $w_{3}^{R(1)}=\frac{1}{3}\left(u_{3}^{N, 1+3}+\pi_{3}^{N}+v_{3}^{N \backslash 2}\right)>$ $w_{3}^{P}$, so $\sigma_{3}^{*}=R(1)$. The resulting equilibrium merger configuration is $1+3$ with probability $\frac{2}{3}$ and $1+2$ with probability $\frac{1}{3}$.

Finally, for $\alpha_{0} \leq \alpha \leq \frac{33}{5}, \max \left\{v_{1}^{N \backslash 3}, u_{1}^{N, 1+2}\right\}>u_{1}^{N, 1+3}$, so $\sigma_{1} \in\{P, R(2)\}$. Given $\sigma_{2}^{*}=R(1)$, we have $\sigma_{3}^{*}=P$ regardless of $\sigma_{1}$. Then, $\sigma_{1}^{*}=R(2)$. The unique equilibrium is $1+2$.

The above analysis shows the general procedure of deriving the equilibrium when there are several profitable mergers. We first determine a firm's optimal choice of target if it proposes a merger using Lemma 4 and similar preferences in other situations. Each firm's strategy then comes down to a choice between passing and proposing to the target. The equilibrium is found by analyzing each firm's best response to various combinations of other firms' strategies. 


\section{Five firms}

$N=\{1,2,3,4,5\}$. If there is any merger, the acquiree must be $2,3,4$ or 5 in the first round. If 2 is acquired (by 1 ) in the first round, we can show (following the analysis of the four-firm case) the further round mergers are $3+4 \Rightarrow 1+5$ for $\alpha \in(43,58]$. Consequently, $\Delta \pi_{1,2}^{N}=v_{1}^{N \backslash 2}-\pi_{1}^{N}-\pi_{2}^{N}=\frac{1}{9}(4 \alpha-45)>0$ in that range of $\alpha$, in which $v_{1}^{N \backslash 2}=v_{1}^{\{1,3,4,5\}}=v_{1}^{\{1,3,5\}}=\frac{1}{2}\left(\pi_{1}^{\{1,3\}}+\pi_{1}^{\{1,3,5\}}-\pi_{5}^{\{1,3,5\}}\right)$. Similarly, if 3 is acquired in the first round, the further round mergers are $2+4 \Rightarrow 1+5$ in $N \backslash 3$ for $\alpha \in(44,59]$. If 4 is acquired in the first round, the further round mergers are $2+3 \Rightarrow 1+5$ for $\alpha \in(44,59]$. The first-round mergers in both cases are also verified to be profitable. If 5 is acquired in the first round, then, as shown in the four-firm case, there will be no further mergers for $\alpha>44$. But given there are no further mergers, the first-round merger is unprofitable for $\alpha>44$.

In summary, for $44<\alpha \leq 59$, there are six merger configurations: (a) $1+2 \Rightarrow 3+4 \Rightarrow 1+5$; (b) $1+3 \Rightarrow 2+4 \Rightarrow 1+5$; (c) $2+3 \Rightarrow 2+4 \Rightarrow 1+5$; (d) $1+4 \Rightarrow 2+3 \Rightarrow 1+5$; (e) $2+4 \Rightarrow 2+3 \Rightarrow 1+5$; (f) $3+4 \Rightarrow 2+3 \Rightarrow 1+5$. All are profitable. ${ }^{18}$ Now consider the firms' preferences of the first-round mergers. In the three first-round mergers in which it would participate [(a), (b) and (d)], firm 1 realizes the largest profit in (d): $u_{1}^{N, 1+4}>u_{1}^{N, 1+3}>u_{1}^{N, 1+2}$. Therefore, $\sigma_{1} \in\{P, R(4)\}$ in the first round. Similarly, we find $\sigma_{2}, \sigma_{3}, \sigma_{4} \in\{P, R(1)\}$. As a result, $\sigma_{1}^{*}=R(4)$. Given this, $\sigma_{2}^{*}=\sigma_{3}^{*}=P$ because $v_{i}^{N}=v_{i}^{N \backslash 4}>u_{i}^{N, 1+i}$ for $i=2,3$. Finally, given 1, 2 and 3's strategies, $\sigma_{4}^{*}=R(1)$.

The above analysis shows that, if there is a first-round merger, there will be no further mergers for $\alpha>59$. We also know that the most profitable single merger in $N$ is $1+5$ with $\alpha_{1,5}^{N}=31.1$. Hence, there is no merger at all for $\alpha>59$, and there is a unique merger configuration of $1+4 \Rightarrow 2+3 \Rightarrow 1+5$ for $44<\alpha \leq 59 .{ }^{19}$

\section{Six firms}

$N=\{1,2,3,4,5,6\}$. We can show that $\alpha_{1}^{N}=38.26, \alpha_{2}^{N}=40.43$, and $\alpha_{3}^{N}=45.57$. If there are four mergers with firm $i$ being acquired in the first round, then the remaining five firms will proceed as in the five-firm case when $\alpha \lesssim \alpha_{3}^{N \backslash i}$. It turns out that $\alpha_{3}^{N \backslash 3}=$

\footnotetext{
${ }^{18}$ When $58<\alpha \leq 59$, (a) is not profitable. But this does not matter, as we show later that it would not be the equilibrium even if it were profitable.

${ }^{19}$ The configuration continues to be the unique equilibrium even when the cost distribution is more general, although the range of $\alpha$ varies depending on the values of the costs.
} 
$\alpha_{3}^{N \backslash 4}=\alpha_{3}^{N \backslash 5}=74$ while $\alpha_{3}^{N \backslash 2}<74$ and $\alpha_{3}^{N \backslash 6}<74$. So when $\alpha>74$, there are no mergers. When $\alpha \lesssim 74$, the only possible configuration involves 3,4 or 5 being acquired in the first round. From now on, assume $\alpha=74$ and focus only on the firms' first-round strategies.

Clearly, $\sigma_{6}^{*}=P$. Analysis of the other firms' preferences leads to $\sigma_{1}, \sigma_{2}, \sigma_{3} \in$ $\{P, R(5)\}$ and $\sigma_{4}, \sigma_{5} \in\{P, R(2)\}$. The acquiree will then be either 4 or 5 . Because $u_{1}^{N, 1+5}<v_{1}^{N \backslash 5}$ (Lemma 3) and $u_{1}^{N, 1+5}<v_{1}^{N \backslash 4}$, we have $\sigma_{1}^{*}=P$. Similarly, $\sigma_{3}^{*}=P$. As a result, the only possible mergers are now $2+4$ and $2+5$ and they are both profitable. Then, $\sigma_{2}^{*}=R(5)$. Given $\sigma_{1}^{*}, \sigma_{2}^{*}, \sigma_{3}^{*}$, we have $w_{4}^{P}=v_{4}^{N \backslash 5}$ regardless of $\sigma_{5}$, and $w_{4}^{R(2)}=\frac{1}{2} u_{4}^{N, 4+2}+\frac{1}{2} v_{4}^{N \backslash 5}$ if $\sigma_{5}=P$ and $w_{4}^{R(2)}=\frac{1}{3} u_{4}^{N, 4+2}+\frac{2}{3} v_{4}^{N \backslash 5}$ if $\sigma_{5}=R(2)$. Furthermore, $u_{4}^{N, 4+2}>v_{4}^{N \backslash 5}$, so $\sigma_{4}^{*}=R(2)$. Finally, $w_{5}^{P}=\frac{1}{2} \pi_{5}^{N}+\frac{1}{2} v_{5}^{N \backslash 4}$ and $w_{5}^{R(2)}=$ $\frac{1}{3}\left(u_{5}^{N, 2+5}+\pi_{5}^{N}+v_{5}^{N \backslash 4}\right)>w_{5}^{P}$, so $\sigma_{5}^{*}=R(2)$. Given the unique equilibrium strategies, the first-round merger is $2+4$ with probability $\frac{1}{3}$ and $2+5$ with probability $\frac{2}{3} .{ }^{20}$

\section{Proposition}

Suppose there are $k(\leq n-2)$ rounds of mergers in $N$. Denote the set of remaining firms after $k$ rounds of mergers as $X \subset N$. Then $x \equiv|X|=n-k$. The last round merger must be $i+j$ for some $i \in X, j \in N \backslash X$ and $j>i$. By Lemma 6, this merger is profitable if and only if

$$
\begin{aligned}
\alpha & <\alpha_{i, j}^{X \cup j}=\frac{(x+2)}{x^{2}-2}\left\{\left(x^{2}+2 x\right) c_{j}-2(x+1) c_{i}\right\}-\left(C_{X}+c_{j}\right) \\
& =\frac{(x+1)^{3}+x+1}{x^{2}-2} c_{j}-\frac{2\left(x^{2}+3 x+2\right)}{x^{2}-2} c_{i}-C_{X} .
\end{aligned}
$$

$\alpha_{i, j}^{X \cup j}$ is maximized when $c_{j}$ is maximized while $c_{i}$ and $C_{X}$ are minimized, which means $i=1, j=n$, and $X$ consists of the first $x$ firms in $N$, hence the expression for $\alpha(k)$ in the proposition.

The construction of $\alpha(k)$ means that a necessary condition for $k$ rounds of mergers is $\alpha<\alpha(k)$. Because $\alpha^{*}=\max _{k} \alpha(k)$, when $\alpha>\alpha^{*}$, there are no mergers; when $\alpha \lesssim \alpha^{*}$, the only possible merger configuration is the one characterized in the Proposition.

\footnotetext{
${ }^{20}$ For general cost distributions, we find that all the above inequalities are valid except that (a) sometimes 3's most preferred target is 2 instead of 5; and (b) sometimes $u_{4}^{N, 4+2}<v_{2}^{N \backslash 5}$. In the first case, $\sigma_{3}^{*}=P$; in the second case, $\sigma_{4}^{*}=P$, leading to a unique first-round merger of $2+5$.
} 


\section{References}

[1] Andrade, G., Mitchell, M., and Stafford, E. "New evidence and perspectives on mergers." Journal of Economic Perspectives, Vol.15 (2001), pp.103-120.

[2] Barros, P.P. "Endogenous mergers and size asymmetry of merger participants." Economics Letters, Vol.60 (1998), pp.113-119.

[3] Cheung, F.K. "Two remarks on the equilibrium analysis of horizontal merger." Economics Letters, Vol.40 (1992), pp.119-123.

[4] Deneckere, R. and Davidson, C. "Incentive to form coalitions with Bertrand competition." Rand Journal of Economics, Vol.16 (1985), pp.473-486.

[5] Dutz, M.A. "Horizontal mergers in declining industries: Theory and evidence." International Journal of Industrial Organization, Vol.7 (1989), pp.11-33.

[6] Farrell, J. and Shapiro, C. "Horizontal mergers: an equilibrium analysis." American Economic Review, Vol.80 (1990), pp.107-26.

[7] Fauli-Oller, R. "Takeover waves." Journal of Economics and Management Strategy, Vol.9 (2000), pp.189-210.

[8] Gowrisankaran, G. "A dynamic model of endogenous horizontal mergers." Rand Journal of Economics, Vol.30 (1999), pp.56-83.

[9] — and Holmes, T.J. "Mergers and the evolution of industry concentration: results from the dominant-firm model." Rand Journal of Economics, Vol.35 (2004), pp.56182.

[10] Hill, K., ed. Cases in Corporate Acquisitions, Buyouts, Mergers, 65 Takeovers. Thomson Gale, 1999.

[11] Horn, H. and Persson, L. "Endogenous mergers in concentrated markets." International Journal of Industrial Organization, Vol.19 (2001), pp.1213-44.

[12] Jensen, M.C. "The modern industrial revolution and the challenge to intern control systems." Journal of Finance, Vol.48 (1993), pp.831-880.

[13] Kamien, M.I. and Zang, I. "The limits of monopolization through acquisition." Quarterly Journal of Economics, Vol.105 (1990), pp.465-99.

[14] Maskin, E. and Tirole, J. "Markov equilibrium." mimeo, Harvard University, 1989. 
[15] Mitchell, M.L. and Mulherin, J.H. "The impact of industry shocks on takeover and restructuring activity." Journal of Financial Studies, Vol.41 (1996), pp.193-229.

[16] Nilssen, T. and Sorgard, L. "Sequential horizontal mergers." European Economic Review, Vol.42 (1998), pp.1683-1702.

[17] Perry, M.K. and Porter, R.H. "Oligopoly and the incentive for horizontal merger." American Economic Review, Vol.75 (1985), pp.219-227.

[18] Salant, S.W., Switzer, S., and Reynolds, R.J. "Losses from horizontal merger: the effects of an exogenous change in industry structure on Cournot-Nash equilibrium." Quarterly Journal of Economics, Vol.98 (1983), pp.185-199.

[19] Thoron, S. "Formation of a coalition-proof stable cartel." Canadian Journal of Economics, Vol.31 (1998), pp.63-76.

[20] United Nations, World Investment Report: Cross-border Mergers and Acquisitions and Development. New York and Geneva, 2000.

[21] White, L.J. "The proposed merger of Coca-Cola and Dr Pepper," in Kwoka, J.E. and White, L.J., eds., The Antitrust Revolution, The Oxford University Press, 1989. 